Tidal dynamics in Kerr spacetime

This article has been downloaded from IOPscience. Please scroll down to see the full text article.

2006 Class. Quantum Grav. 234021

(http://iopscience.iop.org/0264-9381/23/12/002)

View the table of contents for this issue, or go to the journal homepage for more

Download details:

IP Address: 128.206.162.204

The article was downloaded on 30/08/2010 at 16:19

Please note that terms and conditions apply. 


\title{
Tidal dynamics in Kerr spacetime
}

\author{
C Chicone $^{1}$ and B Mashhoon ${ }^{2}$ \\ ${ }^{1}$ Department of Mathematics, University of Missouri-Columbia, Columbia, MO 65211, USA \\ 2 Department of Physics and Astronomy, University of Missouri-Columbia, Columbia, \\ MO 65211, USA
}

Received 21 February 2006

Published 17 May 2006

Online at stacks.iop.org/CQG/23/4021

\begin{abstract}
The motion of free nearby test particles relative to a stable equatorial circular geodesic orbit about a Kerr source is investigated. It is shown that the nonlinear generalized Jacobi equation can be transformed in this case to an autonomous form. Tidal dynamics beyond the critical speed $c / \sqrt{2}$ is studied. We show, in particular, that a free test particle vertically launched from the circular orbit parallel or antiparallel to the Kerr rotation axis is tidally accelerated if its initial relative speed exceeds $c / \sqrt{2}$. Possible applications of our results to high-energy astrophysics are briefly mentioned.
\end{abstract}

PACS number: $04.20 . \mathrm{Cv}$

\section{Introduction}

The purpose of this paper is to study some aspects of the relative motion of free test particles in the gravitational field of a rotating astronomical source. Imagine, for the sake of concreteness, a free mass $m$ in orbit about the central source of mass $M \gg m$ and angular momentum $J$, where $m, M$ and $J$ are constants. Let $\tau$ be the proper time along the orbit of mass $m$ and $\lambda^{\mu}{ }_{(\alpha)}$ be a local orthonormal tetrad system that is parallel transported along this path. Here $\lambda^{\mu}{ }_{(0)}=\mathrm{d} x^{\mu} / \mathrm{d} \tau$ is the four-velocity vector of $m$ and $\lambda^{\mu}{ }_{(i)}, i=1,2,3$, are unit spacelike gyro directions that determine the local spatial frame of $m$. We employ units such that $c=G=1$ throughout; moreover, the signature of the metric is +2 in our convention. Suppose that at $\tau=0$ a probe is launched from $m$ and for some time $\tau>0$ the motion of the probe is closely monitored by observers comoving with $m$. To study relative motion in an invariant manner within the context of general relativity, the quasi-inertial Fermi normal coordinate system [1] is indispensable as it most closely corresponds to actual observations. Let $X^{\alpha}=(T, \mathbf{X})$ be the Fermi coordinates of the probe at an event $P$, then there exists a unique spacelike geodesic of length $\ell$ that connects $P$ orthogonally to the path of $m$ at an event $O$ with proper time $\tau$ such that $T=\tau$ and $X_{i}=\ell n_{\mu} \lambda^{\mu}{ }_{(i)}$, where $n^{\mu}=\left(\mathrm{d} x^{\mu} / \mathrm{d} \ell\right)_{O}$ is the unit vector at $O$ that is tangent to the spacelike geodesic at $O$ and orthogonal to the worldline of $m$. The metric in 
Fermi coordinates is given by

$$
\begin{aligned}
& g_{00}=-1-{ }^{F} R_{0 i 0 j} X^{i} X^{j}+\cdots, \\
& g_{0 i}=-\frac{2}{3}{ }^{F} R_{0 j i k} X^{j} X^{k}+\cdots, \\
& g_{i j}=\delta_{i j}-\frac{1}{3}{ }^{F} R_{i k j l} X^{k} X^{l}+\cdots
\end{aligned}
$$

to second order in the distance away from $m$ that permanently lies at the spatial origin of the Fermi coordinates by construction and has Fermi coordinates $(\tau, \boldsymbol{0})$. Here ${ }^{F} R_{\alpha \beta \gamma \delta}$ is defined by

$$
{ }^{F} R_{\alpha \beta \gamma \delta}=R_{\mu \nu \rho \sigma} \lambda^{\mu}{ }_{(\alpha)} \lambda^{\nu}{ }_{(\beta)} \lambda^{\rho}{ }_{(\gamma)} \lambda^{\sigma}{ }_{(\delta)},
$$

which is the projection of the Riemann curvature tensor on the tetrad frame $\lambda^{\mu}{ }_{(\alpha)}$ along the reference worldline. The theoretical equation of motion of the probe relative to mass $m$ can be described by the reduced geodesic equation [2]

$$
\frac{\mathrm{d}^{2} X^{i}}{\mathrm{~d} T^{2}}+\left(\Gamma_{\alpha \beta}^{i}-\Gamma_{\alpha \beta}^{0} V^{i}\right) \frac{\mathrm{d} X^{\alpha}}{\mathrm{d} T} \frac{\mathrm{d} X^{\beta}}{\mathrm{d} T}=0,
$$

where $\mathbf{V}=\mathrm{d} \mathbf{X} / \mathrm{d} T$ is the Fermi velocity of the probe relative to $m$. The four-velocity of the probe is given by $U^{\mu}=\Gamma(1, \mathbf{V})$, where $\Gamma$ can be determined from

$$
-\frac{1}{\Gamma^{2}}=g_{00}+2 g_{0 i} V^{i}+g_{i j} V^{i} V^{j}<0
$$

since $U^{\mu}$ is a timelike unit vector. The Fermi coordinates are geodesic coordinates based on an orthonormal tetrad frame $\lambda^{\mu}{ }_{(\alpha)}(\tau)$ that is parallel propagated along the orbit of $m$. They are admissible within a cylindrical region of radius $\mathcal{R}_{a}$ along the worldline of $m$; that is, $|\mathbf{X}|<\mathcal{R}_{a}$, where $\mathcal{R}_{a}(T)$ is a certain radius of curvature of spacetime. The nature of $\mathcal{R}_{a}(T)$ has been discussed in detail in our recent paper on explicit Fermi coordinates [3].

For the sake of simplicity, we limit our considerations to $|\mathbf{X}| \ll \mathcal{R}_{a}$. A general feature of the reduced geodesic (5) is that it contains a critical speed given by $1 / \sqrt{2}$, especially in the case of one-dimensional motion. The notion of critical speed in gravitational motion has been recently reviewed in [4]. We are particularly interested in the role that the critical speed $V_{c}=1 / \sqrt{2}$ plays in the motion of the probe; therefore the probe can be launched from $m$ at any initial speed $V_{0}<1$, but-for simplicity-we concentrate on its motion only when it is relatively close to the reference particle $m$. Equation (5) to first order in $|\mathbf{X}|$ is the generalized Jacobi equation [2]

$$
\begin{aligned}
& \frac{\mathrm{d}^{2} X^{i}}{\mathrm{~d} T^{2}}+{ }^{F} R_{0 i 0 j} X^{j}+2{ }^{F} R_{i k j 0} V^{k} X^{j}+\frac{2}{3}\left(3^{F} R_{0 k j 0} V^{i} X^{k}\right. \\
&\left.+{ }^{F} R_{i k j \ell} V^{k} V^{\ell}+{ }^{F} R_{0 k j \ell} V^{i} V^{k} V^{\ell}\right) X^{j}=0,
\end{aligned}
$$

which describes the timelike motion of the probe when

$$
\frac{1}{\Gamma^{2}}=1-V^{2}+{ }^{F} R_{0 i 0 j} X^{i} X^{j}+\frac{4}{3}{ }^{F} R_{0 j i k} X^{j} V^{i} X^{k}+\frac{1}{3}{ }^{F} R_{i k j \ell} V^{i} X^{k} V^{j} X^{\ell}>0 .
$$

For $|\mathbf{V}| \ll 1$, we can drop all velocity-dependent terms in equations (7) and (8); then, the inequality in (8) is satisfied and (7) reduces to the Jacobi equation

$$
\frac{\mathrm{d}^{2} X^{i}}{\mathrm{~d} T^{2}}+{ }^{F} R_{0 i 0 j} X^{j}=0
$$

Suppose that the worldline of the probe can be determined in the Fermi coordinate system on the basis of equations (5) and (6); then, it is in principle possible to find the representation of 
this worldline in the background coordinate system using the explicit coordinate transformation between the two systems of coordinates. In practice, however, it is not possible in general to find explicit expressions for Fermi coordinates in terms of the background coordinates and therefore one has to resort to approximation schemes; see [3] for a general discussion of this problem. Nevertheless, it is important to recognize that once $\mathbf{X}(T)$ is determined from the generalized Jacobi equation (or the Jacobi equation), the worldline of the probe is completely characterized. That is, if in the background coordinate system the worldline of $m$ is given by $x^{\mu}(\tau)$, then the worldline of the probe is $x_{p}^{\mu}(\tau)$, where

$$
x_{p}^{\mu}(\tau)=x^{\mu}(\tau)+X^{i}(\tau) \lambda_{(i)}^{\mu}(\tau)
$$

and we have replaced the Fermi temporal coordinate $T$ by $\tau$ in the $\operatorname{argument}$ of $\mathbf{X}$. This relation follows from the definition of Fermi coordinates and the fact that $x_{p}^{\mu}(\tau)-x^{\mu}(\tau) \approx \ell n^{\mu}$ for $\ell \ll \mathcal{R}_{a}$, since the (generalized) Jacobi equation is linear in the distance away from the reference trajectory. Alternatively, $x_{p}^{\mu}(\tau)$ may be viewed as a perturbed geodesic orbit. Depending upon whether $\mathbf{X}$ is determined from the Jacobi or the generalized Jacobi equation, the results of this approach may be interpreted respectively in terms of the linear or nonlinear perturbations of geodesic orbits in the background gravitational field.

Regarding the source of the gravitational field and the reference trajectory, we assume that $m$ is on a stable equatorial circular geodesic orbit about a Kerr source. This choice for the orbit to some extent complements our previous work on the motion of astrophysical jets $[5,6]$, where the reference trajectory is a radial geodesic along the rotation axis of a Kerr source. The geodesics of Kerr spacetime have been discussed by a number of authors; see, for instance, [7] and references therein. The characteristics of the source and the reference trajectory will be described in detail in the next section. Various aspects of tidal dynamics in black-hole spacetimes have been discussed in a number of papers; see, for instance, [8-12] and the references cited therein. Furthermore, we treat free-particle orbits for the sake of simplicity; a corresponding discussion of black-hole accretion discs [13] is beyond the scope of this investigation. Section 3 is devoted to the solution of the Jacobi equation. We show in section 4 that a rotation introduced in section 3 leads to a complete transformation of the generalized Jacobi equation to an autonomous form. This equation is then analysed in detail. Finally, section 5 contains a brief discussion of our results.

\section{Reference worldline}

The spacetime region of interest is the exterior Kerr domain with the metric

$-\mathrm{d} s^{2}=-\mathrm{d} t^{2}+\frac{\Sigma}{\Delta} \mathrm{d} r^{2}+\Sigma \mathrm{d} \theta^{2}+\left(r^{2}+a^{2}\right) \sin ^{2} \theta \mathrm{d} \phi^{2}+\frac{2 M r}{\Sigma}\left(\mathrm{d} t-a \sin ^{2} \theta \mathrm{d} \phi\right)^{2}$,

where $a=J / M$ is the specific angular momentum of the source. Here $(t, r, \theta, \phi)$ are the standard Boyer-Lindquist coordinates and

$$
\Sigma=r^{2}+a^{2} \cos ^{2} \theta, \quad \Delta=r^{2}-2 M r+a^{2}
$$

The reference trajectory is assumed to be a stable circular orbit of fixed radius $r$ in the equatorial plane $\theta=\pi / 2$; in terms of $r$, such orbits exist from infinity all the way down to the last stable circular orbits given by

$$
1-\frac{6 M}{r} \pm 8 a \sqrt{\frac{M}{r^{3}}}-3 \frac{a^{2}}{r^{2}}=0 .
$$


Throughout this paper, the upper (lower) sign refers to orbits where $m$ rotates in the same (opposite) sense as the source. For $r$ less than the solution of (13), there are unstable circular orbits that end at the null circular orbits given by

$$
1-\frac{3 M}{r} \pm 2 a \sqrt{\frac{M}{r^{3}}}=0
$$

It is useful to define the Keplerian frequency for the orbits under consideration here as

$$
\omega_{K}= \pm \sqrt{\frac{M}{r^{3}}}
$$

Let us note that in the orbital equations (13) and (14), a prograde orbit becomes retrograde and vice versa when $a \rightarrow-a$; this circumstance explains why the combination $a \omega_{k}$ usually appears in orbital equations. We assume that $a \geqslant 0$ throughout; therefore, the sign of $\omega_{K}$ indicates the sense of the orbit. It follows from the geodesic equation that along the reference worldline

$$
t=\frac{1+a \omega_{K}}{N} \tau, \quad \phi=\frac{\omega_{K}}{N} \tau
$$

where

$$
N=\sqrt{1-\frac{3 M}{r}+2 a \omega_{K}}
$$

is such that the null circular orbits are solutions of $N=0$ and we have assumed in (16) that $t=\tau=0$ at $\phi=0$. The constants of the motion for the reference worldline are the specific energy $E$ and orbital angular momentum $L$, associated respectively with the timelike and azimuthal Killing vectors $\partial_{t}$ and $\partial_{\phi}$, and are given by

$$
E=\frac{1}{N}\left(1-\frac{2 M}{r}+a \omega_{K}\right), \quad L=\frac{r^{2} \omega_{K}}{N}\left(1-2 a \omega_{K}+\frac{a^{2}}{r^{2}}\right) .
$$

To determine the orthonormal tetrad $\lambda^{\mu}{ }_{(\alpha)}$, let us first consider the tetrad $\Lambda_{(\alpha)}^{\mu}$ carried by the fundamental static observers in the exterior Kerr spacetime. In the equatorial plane and in terms of $(t, r, \theta, \phi)$, the natural orthonormal tetrad of these observers along the coordinate directions is given by

$$
\begin{array}{ll}
\Lambda_{(0)}^{\mu}=\left(\frac{1}{A}, 0,0,0\right), & \Lambda_{(1)}^{\mu}=\left(0, \frac{\sqrt{\Delta}}{r}, 0,0\right), \\
\Lambda_{(2)}^{\mu}=\left(0,0, \frac{1}{r}, 0\right), & \Lambda_{(3)}^{\mu}=\left(-\frac{2 M a}{r A \sqrt{\Delta}}, 0,0, \frac{A}{\sqrt{\Delta}}\right),
\end{array}
$$

where

$$
A=\sqrt{1-\frac{2 M}{r}}
$$

Next, we subject this tetrad to a Lorentz boost with speed $\tilde{\beta}, \Lambda^{\mu}{ }_{(\alpha)} \rightarrow \tilde{\Lambda}_{(\alpha)}^{\mu}$, such that $\tilde{\Lambda}^{\mu}{ }_{(0)}=\lambda^{\mu}(0)$ is the unit vector tangent to the worldline of the reference particle $m$. The new orthonormal tetrad along the orbit of $m$ is

$$
\begin{aligned}
& \tilde{\Lambda}_{(0)}^{\mu}=\tilde{\gamma}\left[\Lambda_{(0)}^{\mu}+\tilde{\beta} \Lambda_{(3)}^{\mu}\right], \quad \tilde{\Lambda}_{(1)}^{\mu}=\Lambda_{(1)}^{\mu}, \\
& \tilde{\Lambda}_{(2)}^{\mu}=\Lambda_{(2)}^{\mu}, \quad \tilde{\Lambda}_{(3)}^{\mu}=\tilde{\gamma}\left[\Lambda_{(3)}^{\mu}+\tilde{\beta} \Lambda_{(0)}^{\mu}\right],
\end{aligned}
$$




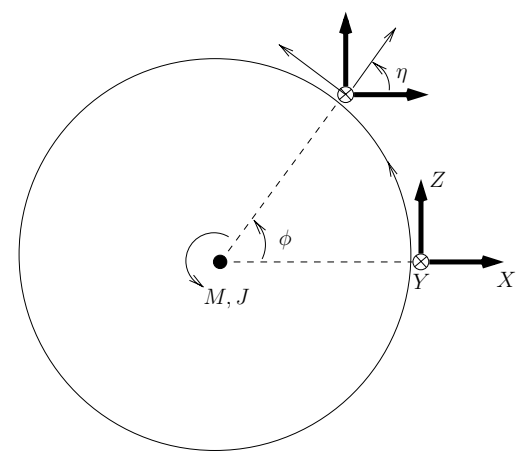

Figure 1. Schematic diagram that depicts the parallel transport of the spatial triad along a prograde circular orbit.

where $\tilde{\gamma}$ is the Lorentz factor corresponding to $\tilde{\beta}$. We find from $\tilde{\Lambda}^{\mu}(0)=\lambda^{\mu}(0)$ that the Lorentz pair $(\tilde{\beta}, \tilde{\gamma})$ is given by

$$
\tilde{\beta}=\frac{\sqrt{\Delta} \omega_{K}}{E N}, \quad \tilde{\gamma}=\frac{E}{A} .
$$

Clearly, $\tilde{\beta}$ is positive (negative) for prograde (retrograde) orbits; moreover, $\tilde{\gamma}$ diverges at the null orbits $(N=0)$. The spatial triad of $\tilde{\Lambda}_{(\alpha)}^{\mu}$ is along the spherical polar coordinate directions, which are the radial, normal and tangential directions with respect to the orbit, and therefore needs to be rotated back such that the resulting tetrad would then be parallel propagated along the orbit as illustrated in figure 1. Thus

$$
\begin{array}{ll}
\lambda^{\mu}{ }_{(0)}=\tilde{\Lambda}_{(0)}^{\mu}, & \lambda_{(1)}^{\mu}=\tilde{\Lambda}_{(1)}^{\mu} \cos \eta-\tilde{\Lambda}_{(3)}^{\mu} \sin \eta, \\
\lambda^{\mu}{ }_{(2)}=\tilde{\Lambda}_{(2)}^{\mu}, & \lambda_{(3)}^{\mu}=\tilde{\Lambda}_{(1)}^{\mu} \sin \eta+\tilde{\Lambda}_{(3)}^{\mu} \cos \eta .
\end{array}
$$

It follows from the vanishing of the covariant derivative of $\lambda^{\mu}{ }_{(i)}$ along the orbit that

$$
\eta=\omega_{K} \tau,
$$

where we have chosen the integration constant such that $\eta=0$ at $\tau=0$. Note that $\phi-\eta=\left(N^{-1}-1\right) \eta$, which, to first order in $M / r \ll 1$ and $a / r \ll 1$ can be written as

$$
\phi-\eta \approx\left(\frac{3}{2} \frac{M}{r} \omega_{K}-\frac{J}{r^{3}}\right) \tau
$$

that is, the difference between these angles is due to a combination of geodetic and gravitomagnetic precessions. The final result for the tetrad frame in $(t, r, \theta, \phi)$ coordinates is then

$$
\begin{aligned}
\lambda^{\mu}{ }_{(0)} & =\frac{1}{N}\left(1+a \omega_{K}, 0,0, \omega_{K}\right), \\
\lambda^{\mu}{ }_{(1)} & =\frac{1}{\sqrt{\Delta}}\left(-L \sin \eta, \frac{\Delta}{r} \cos \eta, 0,-E \sin \eta\right), \\
\lambda_{(2)}^{\mu} & =\left(0,0, \frac{1}{r}, 0\right),
\end{aligned}
$$




$$
\lambda_{(3)}^{\mu}=\frac{1}{\sqrt{\Delta}}\left(L \cos \eta, \frac{\Delta}{r} \sin \eta, 0, E \cos \eta\right) .
$$

Finally, we need to compute the projection of the Riemann tensor along the orbit on this tetrad frame as in equation (4). The result may be expressed as a $6 \times 6$ matrix $\left(\mathcal{R}_{A B}\right)$, where $A$ and $B$ are indices that belong to the set $\{01,02,03,23,31,12\}$. This matrix has the form

$$
\mathcal{R}=\left[\begin{array}{cc}
\mathcal{E} & \mathcal{H} \\
\mathcal{H} & -\mathcal{E}
\end{array}\right],
$$

where $\mathcal{E}$ and $\mathcal{H}$ are $3 \times 3$ symmetric and traceless matrices containing respectively the electric and magnetic components of the Riemann tensor. It turns out that in the case under consideration

$$
\mathcal{E}=\kappa\left[\begin{array}{ccc}
k_{1} & 0 & k^{\prime} \\
0 & k_{2} & 0 \\
k^{\prime} & 0 & k_{3}
\end{array}\right], \quad \mathcal{H}=\kappa\left[\begin{array}{ccc}
0 & h & 0 \\
h & 0 & h^{\prime} \\
0 & h^{\prime} & 0
\end{array}\right]
$$

where

$$
\kappa=\omega_{K}^{2}=\frac{M}{r^{3}} .
$$

The proportionality $\mathcal{R} \propto \kappa$ is expected on the basis of the correspondence with Newtonian tides. In (34), $k_{2}=-\left(k_{1}+k_{3}\right)$ is constant and is given by $k_{2}=3 \gamma^{2}-2$, while

$k_{1}=1-3 \gamma^{2} \cos ^{2} \eta, \quad k_{3}=1-3 \gamma^{2} \sin ^{2} \eta, \quad k^{\prime}=-3 \gamma^{2} \sin \eta \cos \eta$,

$h=-3 \gamma^{2} \beta \cos \eta, \quad h^{\prime}=-3 \gamma^{2} \beta \sin \eta$.

Here $(\beta, \gamma)$ is a new Lorentz pair, i.e. $\gamma=1 / \sqrt{1-\beta^{2}}$, given by

$$
\beta=\frac{r^{2} \omega_{K}-a}{\sqrt{\Delta}}, \quad \gamma=\frac{\sqrt{\Delta}}{r N},
$$

which reduces to $(\tilde{\beta}, \tilde{\gamma})$ in the Schwarzschild limit $(a=0)$; moreover, $\gamma$ diverges at the null orbits $(N=0)$.

Let us note that for the orbits under consideration and $a \leqslant M, \beta \in[-1 / 2,1 / 2]$; that is, $\beta$ is positive (negative) for a prograde (retrograde) orbit and far from the source $(r \gg 2 M)$, $|\beta| \sim \sqrt{M / r}$, so that $\beta \rightarrow 0$ as $r \rightarrow \infty$, then $|\beta|$ monotonically increases from zero to $1 / 2$ at the last stable circular orbits. Furthermore, for $a>M, \beta$ is always negative for retrograde orbits, but it is not always positive for prograde orbits; in fact, $\beta$ vanishes for a prograde orbit with radius $r=a^{2} / M$.

These curvature components can now be used in equations (7) and (9) to study the motion of the probe relative to $m$. It should be mentioned that our results for the tidal tensor $\mathcal{E}$ are consistent with the work of Marck [9].

\section{Jacobi equation}

Let us now turn to the solution of the Jacobi equation (9). For simplicity, we use instead of the Fermi coordinates $X^{\alpha}=(T, \mathbf{X})$, the dimensionless Fermi coordinates $(\eta, \mathbf{x})$,

$$
\omega_{K} X^{\alpha}:=(\eta, \mathbf{x})
$$


where $\mathbf{x}=(x, y, z)$. The Jacobi equation then takes the form

$$
\begin{array}{ll}
\frac{\mathrm{d}^{2}}{\mathrm{~d} \eta^{2}}\left[\begin{array}{l}
x \\
z
\end{array}\right]=S\left[\begin{array}{l}
x \\
z
\end{array}\right], & S=\left[\begin{array}{ll}
3 \gamma^{2} \cos ^{2} \eta-1 & 3 \gamma^{2} \sin \eta \cos \eta \\
3 \gamma^{2} \sin \eta \cos \eta & 3 \gamma^{2} \sin ^{2} \eta-1
\end{array}\right], \\
\frac{\mathrm{d}^{2} y}{\mathrm{~d} \eta^{2}}+\sigma^{2} y=0, & \sigma=\sqrt{3 \gamma^{2}-2} .
\end{array}
$$

Thus normal to the equatorial plane, we have a simple harmonic motion of frequency $\left|\omega_{K}\right| \sigma$, where $\sigma$ ranges from 1 to $\sqrt{2}$ for $a \leqslant M$; that is, it increases monotonically from unity at $r=\infty$ to $\sqrt{2}$ at the last stable circular orbits. The equations of relative motion in the equatorial plane can be put into an autonomous form by a rotation to the radial and tangential directions (cf figure 1). That is, let

$$
\left[\begin{array}{l}
\xi \\
\zeta
\end{array}\right]=R\left[\begin{array}{l}
x \\
z
\end{array}\right], \quad R=\left[\begin{array}{cc}
\cos \eta & \sin \eta \\
-\sin \eta & \cos \eta
\end{array}\right]
$$

Then, equation (40) reduces to the autonomous system

$$
\ddot{\xi}-2 \dot{\zeta}-3 \gamma^{2} \xi=0, \quad \ddot{\zeta}+2 \dot{\xi}=0
$$

where an overdot denotes differentiation with respect to $\eta$. Assuming that at $\eta=0, \mathbf{x}=0$ and the initial velocity of the probe $\left(V_{0} \ll 1\right)$ has components

$$
\mathbf{V}_{\mathbf{0}}=V_{0}(\sin \vartheta \cos \varphi, \cos \vartheta, \sin \vartheta \sin \varphi)
$$

in the local $(X, Y, Z)$ system, we find

$$
\begin{aligned}
& \xi=\frac{V_{0} \sin \vartheta}{\lambda^{2}}[2(1-\cos \lambda \eta) \sin \varphi+\lambda \sin \lambda \eta \cos \varphi], \\
& y=\frac{1}{\sigma} V_{0} \cos \vartheta \sin \sigma \eta, \\
& \zeta=-\frac{V_{0} \sin \vartheta}{\lambda^{2}}\left[\left(3 \gamma^{2} \eta-\frac{4}{\lambda} \sin \lambda \eta\right) \sin \varphi+2(1-\cos \lambda \eta) \cos \varphi\right],
\end{aligned}
$$

where $\lambda=\sqrt{4-3 \gamma^{2}}$. We note that $\lambda \geqslant 0$, since

$$
\lambda^{2}=\frac{1}{N^{2}}\left(1-6 \frac{M}{r}+8 a \omega_{K}-3 \frac{a^{2}}{r^{2}}\right),
$$

so that for $a \leqslant M, \lambda$ ranges from zero to unity; that is, it decreases monotonically from unity at $r=\infty$ to zero at the last stable circular orbits. If the reference trajectory is one of the last stable circular orbits, then $\gamma^{2}=4 / 3, y$ is given by (46) with $\sigma=\sqrt{2}$ and

$$
\begin{aligned}
& \xi=V_{0} \sin \vartheta\left(\eta \cos \varphi+\eta^{2} \sin \varphi\right), \\
& \zeta=V_{0} \sin \vartheta\left[\left(\eta-\frac{2}{3} \eta^{3}\right) \sin \varphi-\eta^{2} \cos \varphi\right] .
\end{aligned}
$$

Recalling the restrictions $|\mathbf{X}| \ll \mathcal{R}_{a}$ and $|\mathbf{V}| \ll 1$, which in this case translate to $|\mathbf{x}| \ll 1$ and $|\dot{\mathbf{x}}| \ll 1$, we find that the validity of equations (47), (49) and (50) is limited in time due to the presence of secular terms.

It is interesting to note that terms that appear in the solution of the Jacobi equation in equations (45) and (47) with proper frequency $\lambda\left|\omega_{K}\right|$ have frequency $\lambda\left|\omega_{K}\right| N /\left(1+a \omega_{K}\right)$ with respect to the coordinate time $t$; to lowest order in $M / r \ll 1$ and with $a=0$, the deviation of this frequency from the Keplerian frequency corresponds to the Einstein precession frequency 
for an orbit of vanishing eccentricity, while the first-order correction in $a / r \ll 1$ corresponds to the de Sitter-Lense-Thirring precession frequency [14, 15].

Finally, a remark is in order here regarding the boundary condition that $\mathbf{X}=0$ at $\tau=0$, which corresponds to our assumption that the probe is initially launched from $m$. In fact, it is only necessary that the probe be near the reference particle $m$, so that $|\mathbf{X}|$ must be initially very small compared to $\mathcal{R}_{a}$; for the sake of simplicity, we choose $|\mathbf{X}(0)|=0$ throughout this paper. This means that in terms of $(\xi, y, \zeta)$, we always assume that

$$
\left.(\xi, y, \zeta)\right|_{\eta=0}=0,\left.\quad(\dot{\xi}, \dot{y}, \dot{\zeta})\right|_{\eta=0}=\mathbf{V}_{0}
$$

\section{Generalized Jacobi equation}

The reduction of the Jacobi equation to the autonomous system (43) is basically due to the fact that $\operatorname{RSR}^{-1}=D$, where $D=\operatorname{diag}\left(3 \gamma^{2}-1,-1\right)$ is a constant matrix. System (40) then takes the form

$$
\left[\begin{array}{lll}
\ddot{\xi} & -2 \dot{\zeta} & -\xi \\
\ddot{\zeta} & +2 \dot{\xi} & -\zeta
\end{array}\right]=D\left[\begin{array}{l}
\xi \\
\zeta
\end{array}\right],
$$

which is equivalent to system (43). The transformation from $(x, z)$ to $(\xi, \zeta)$ involves a rotation from a local inertial frame to rotating axes; therefore, in equation (52) we note the presence of Coriolis and centripetal terms on the left-hand side of this system. Moreover, this transformation is related to Hill's contributions to the classical three-body problem [16, 17]; indeed, system (43) in the Newtonian limit $\gamma \rightarrow 1$ is equivalent to a limiting form of the Hill system discussed in [16], namely, system (41) of [16] with $k=e_{1}=0$.

It can be shown, after much algebra, that under the same rotation as in equation (42), the nonlinear generalized Jacobi equation (7) can be rendered autonomous as well. This remarkable fact is due to the special symmetries of the Kerr spacetime [18]. Introducing $\Delta_{\xi}=\dot{\xi}-\zeta$ and $\Delta_{\zeta}=\dot{\zeta}+\xi$, where

$$
\left[\begin{array}{c}
\Delta_{\xi} \\
\Delta_{\zeta}
\end{array}\right]=R\left[\begin{array}{c}
\dot{x} \\
\dot{z}
\end{array}\right],
$$

we find that $x \dot{x}+z \dot{z}=\xi \Delta_{\xi}+\zeta \Delta_{\zeta}, x \dot{z}-z \dot{x}=\xi \Delta_{\zeta}-\zeta \Delta_{\xi}$, and

$$
\begin{aligned}
& \ddot{\xi}-2 \dot{\zeta}-3 \gamma^{2} \xi=2 \Delta_{\xi}\left[\zeta \Delta_{\zeta}-\left(\sigma^{2}+1\right) \xi \Delta_{\xi}\right]+6 \gamma^{2} \beta \xi \Delta_{\zeta} \\
&-2\left(\gamma^{2} \beta \Delta_{\xi}^{2}-\frac{1}{3} \sigma^{2} \Delta_{\zeta}\right)\left(\xi \Delta_{\zeta}-\zeta \Delta_{\xi}\right)+2 y \dot{y} \Delta_{\xi}\left(\gamma^{2} \beta \Delta_{\zeta}+\sigma^{2}-\frac{1}{3}\right) \\
&-2 \dot{y}^{2}\left(\gamma^{2} \beta \zeta \Delta_{\xi}-\frac{1}{3} \xi\right), \\
& \ddot{y}+\sigma^{2}\left(1-2 \dot{y}^{2}\right) y=\frac{2}{3} y\left[\Delta_{\xi}^{2}-\left(\sigma^{2}+1\right) \Delta_{\zeta}^{2}-9 \gamma^{2} \beta \Delta_{\zeta}\right]-2 \dot{y}\left[\gamma^{2} \beta \Delta_{\xi}\left(\xi \Delta_{\zeta}-\zeta \Delta_{\xi}\right)\right. \\
&\left.+\left(3 \gamma^{2}-\frac{2}{3}\right) \xi \Delta_{\xi}-\left(\gamma^{2}+\frac{2}{3}\right) \zeta \Delta_{\zeta}\right]+2 \gamma^{2} \beta \dot{y}^{2}\left(y \Delta_{\zeta}-\zeta \dot{y}\right), \\
& \ddot{\zeta}+2 \dot{\xi}=2 \Delta_{\zeta}\left[\zeta \Delta_{\zeta}-\left(\sigma^{2}+1\right) \xi \Delta_{\xi}\right]-6 \gamma^{2} \beta \xi \Delta_{\xi}-2 \Delta_{\xi}\left(\gamma^{2} \beta \Delta_{\zeta}+\frac{1}{3} \sigma^{2}\right)\left(\xi \Delta_{\zeta}-\zeta \Delta_{\xi}\right) \\
&+ \frac{2}{3}\left(\sigma^{2}+1\right) \dot{y}\left(y \Delta_{\zeta}-\zeta \dot{y}\right)+2 y \dot{y}\left[\gamma^{2} \beta\left(\Delta_{\zeta}^{2}+3\right)+\sigma^{2} \Delta_{\zeta}\right]-2 \gamma^{2} \beta \dot{y}^{2} \zeta \Delta_{\zeta} .
\end{aligned}
$$

Starting with the exact solution of the Jacobi equation as the unperturbed solution, it is straightforward to develop a solution of equations (54)-(56) via the standard perturbation expansion in terms of $V_{0} \ll 1$. However, the results of such a perturbation scheme, based on the small parameter $V_{0}$, should be used only in conjunction with the higher-order tidal terms - that is, terms that have been neglected in the expansions of the gravitational potentials 
in equations (1)-(3) and would result in terms in the equation of motion (5) that go beyond the linear order in $|\mathbf{X}|$.

It follows from equations (10), (29)-(32), (39) and (42) that the trajectory of the probe in Boyer-Lindquist coordinates, $x_{p}^{\mu}=\left(t_{p}, r_{p}, \theta_{p}, \phi_{p}\right)$, is given by

$$
\begin{aligned}
& t_{p}(\tau)=\frac{1}{N}\left(1+a \omega_{K}\right) \tau+\frac{L}{\sqrt{\Delta} \omega_{K}} \zeta, \\
& r_{p}(\tau)=r+\frac{\sqrt{\Delta}}{r \omega_{K}} \xi, \\
& \theta_{p}(\tau)=\frac{\pi}{2}+\frac{1}{r \omega_{K}} y, \\
& \phi_{p}(\tau)=\frac{1}{N} \omega_{K} \tau+\frac{E}{\sqrt{\Delta} \omega_{K}} \zeta .
\end{aligned}
$$

Using $\xi(\eta), y(\eta)$ and $\zeta(\eta)$ from the (generalized) Jacobi equation and replacing $\eta=\omega_{K} T$ by $\omega_{K} \tau$, we can determine the path of the probe in the standard Kerr coordinate system. This is due to the fact that the generalized Jacobi equation is linear in the distance $|\mathbf{X}|$ away from the reference geodesic; therefore, $X^{i} \lambda^{\mu}{ }_{(i)}$ may be viewed as a generalized Jacobi field that is defined along the reference geodesic. It turns out that for small-amplitude perturbations $\left(V_{0} \ll 1\right)$, the radial $(\xi)$ and vertical $(y)$ motions in general contain the basic proper epicyclic frequencies $\lambda\left|\omega_{K}\right|$ and $\sigma\left|\omega_{K}\right|$, respectively.

The autonomous system of equations (54)-(56) naturally splits into equations for the vertical and equatorial motions. That is, if the probe is launched vertically in the $y$ direction, then its motion will be confined to the $y$ direction according to the generalized Jacobi equation. Similarly, if the probe is launched in the orbital $(\xi, \zeta)$ plane, then the probe will remain in this plane throughout its motion.

\subsection{Vertical motion}

If the probe is launched in the purely vertical direction relative to the reference orbit, then $\xi(\eta)=\zeta(\eta)=0$ for all $\eta$ and hence the equations of motion reduce to

$$
\ddot{y}+\sigma^{2}\left(1-2 \dot{y}^{2}\right) y=0 .
$$

The vertical motion is simply uniform for $\dot{y}= \pm 1 / \sqrt{2}$; that is, equation (61) exhibits the critical speed $V_{c}=1 / \sqrt{2}$ beyond which the character of the motion is opposite to the lowspeed limit that agrees with Newtonian expectations. If the probe is launched with $V_{0}<V_{c}$, then it will begin to decelerate in agreement with Newtonian gravity. However, for $V_{0}>V_{c}$, the probe will accelerate, as treated in detail in $[2,5]$. In fact, the vertical motion can be described exactly $[2,4,5]$, since equation (61) implies that

$$
\dot{y}^{2}=V_{c}^{2}+\left(V_{0}^{2}-V_{c}^{2}\right) \mathrm{e}^{2 \sigma^{2} y^{2}}
$$

whose solution can be determined by quadrature. This solution represents timelike geodesic motion if the following condition is satisfied

$$
\frac{1}{\Gamma^{2}}=1-\dot{y}^{2}+\sigma^{2} y^{2}>0
$$

For $V_{0}^{2}<V_{c}^{2}$, condition (63) is always satisfied and it follows from (62) that the motion of $y$ is periodic and confined to the interval $\left[-y_{\max }, y_{\max }\right]$, where

$$
y_{\max }=\frac{1}{\sigma} \sqrt{\frac{1}{2} \ln \left(\frac{V_{c}^{2}}{V_{c}^{2}-V_{0}^{2}}\right)} .
$$


On the other hand, for $V_{0}^{2}>V_{c}^{2}$ the particle is accelerated to (almost) the local speed of light. In this way, the particle gains enormous tidal energy. According to condition (63), the range of $y$ over which this happens monotonically shrinks to zero as $V_{0}$ approaches unity. The question of the admissibility of Fermi coordinates in such a case is a difficult one; an unsuccessful attempt to answer such a question is contained in our recent work [3]. It is essential-for astrophysical applications-to connect these local results to what distant observers would measure [5]. Indeed, the gravitational tidal energy of particles within the Fermi coordinate system can be transferred to the outside world (i.e. beyond the Fermi system) through collisions with other particles or emission of radiation.

In our previous work involving tidal dynamics about a reference escape trajectory along the axis of rotation of the central source [5], we found that for relative speeds above the critical speed $V_{c}$, tidal deceleration occurs in a cone of angle $\Theta$ measured from the rotation axis, where $\Theta$ is given by $\tan \Theta=1 / V_{c}$ and corresponds to an angle of about $55^{\circ}$, while tidal acceleration occurs outside this cone, i.e. within the complement of the critical-velocity cone involving latitudes from $-\Theta^{\prime}$ to $\Theta^{\prime}$, where $\Theta^{\prime}=\pi / 2-\Theta$ and corresponds to an angle of about $35^{\circ}$. On the basis of these previous results, we expect that in the present situation the phenomenon of tidal acceleration for speeds above the critical speed may not be restricted to the vertical axis alone. To investigate this issue in the case under consideration, we have integrated the full system of equations (54)-(56) with initial conditions (51). Our results indicate that vertical acceleration-either parallel or antiparallel to the Kerr rotation axis-occurs when the probe is launched with $V_{0}>1 / \sqrt{2}$ in a direction $(\vartheta, \varphi)$, where for a given $\varphi \in[0,2 \pi), \vartheta$ is restricted to a certain interval, namely, $\vartheta \in\left[0, \vartheta_{0}\right)$. To clarify the situation analytically, let us first note that if $(\xi, y, \zeta)$ is a solution of equations $(54)-(56)$, then so is $(\xi,-y, \zeta)$. In particular, equation (61) is clearly invariant under the parity transformation $y \mapsto-y$; therefore, vertical motion is expected to occur symmetrically with respect to the equatorial plane. For this reason, we limit our considerations to $y>0$, which corresponds to motion antiparallel to the Kerr axis in our convention (see figure 1). Furthermore, let us note that with initial conditions (51), vertical acceleration vanishes initially, i.e. $\ddot{y}(\eta)=0$ at $\eta=0$; therefore, it proves useful to compute $\dddot{y}(0)$ using equation (55). It turns out that

$$
\dddot{y}(0)=2 V_{0} W(\vartheta, \varphi) \cos \vartheta,
$$

where $W$ is a quadratic function of $\rho=\sin \vartheta$ given by

$$
W=C_{0}-V_{0} C_{1} \rho-V_{0}^{2} C_{2} \rho^{2} .
$$

Here $C_{0}, C_{1}$ and $C_{2}$ are defined by

$$
\begin{aligned}
& C_{0}=\sigma^{2}\left(V_{0}^{2}-\frac{1}{2}\right), \quad C_{1}=3 \gamma^{2} \beta \sin \varphi, \\
& C_{2}=\left(2 \sigma^{2}+1\right)-\left(\sigma^{2}+2\right) \sin ^{2} \varphi .
\end{aligned}
$$

For $\vartheta=0, \dddot{y}(0)$ is positive for $V_{0}>1 / \sqrt{2}$; in fact, this is the case for an extended domain about the Kerr axis. The boundary of this domain is characterized by $W=0$, i.e. $\rho(\varphi)=\sin \vartheta_{0}$ given by

$$
\rho(\varphi)=\frac{1}{2 V_{0} C_{2}}\left(\sqrt{C_{1}^{2}+4 C_{0} C_{2}}-C_{1}\right),
$$

and $\rho=1$ (i.e. $\vartheta_{0}=\pi / 2$ ), where $\dddot{y}(0)$ vanishes, as illustrated in figure 2 , where $V_{0}=0.8$ and $\beta=0.45$. Let us recall that for a prograde reference orbit of radius $r$ about a Kerr black hole, $\beta$ increases monotonically from zero at $r=\infty$ to 0.5 at the last stable circular orbit. For a fixed $\beta \in(0,0.5]$ and $V_{0}>V_{c}$, the tidal-acceleration domain expands as $V_{0} \rightarrow 1$. 


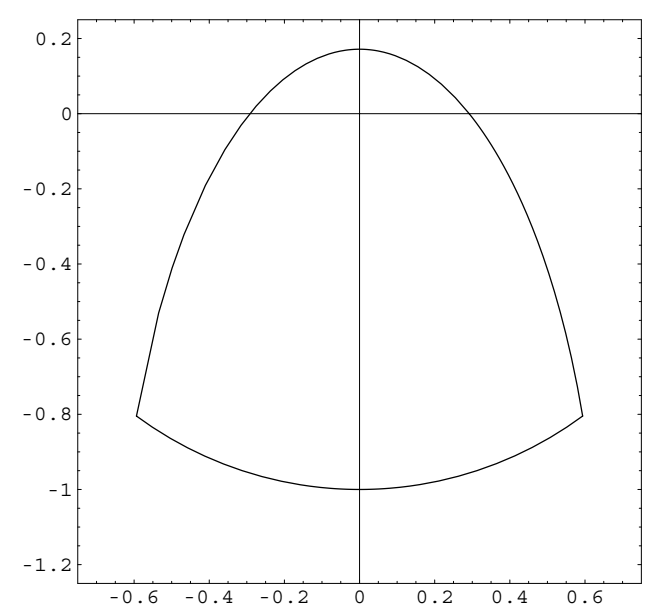

Figure 2. Plot of the boundary of the domain - in the $(\hat{x}, \hat{y})$ plane-in which the vertical tidal acceleration of the probe is positive for $V_{0}>1 / \sqrt{2}$. The probe is launched in the direction $(\vartheta, \varphi)$ with respect to the local frame of the reference observer. We define $\hat{x}:=\rho \cos \varphi, \hat{y}:=\rho \sin \varphi$, where $\rho=\sin \vartheta$. For this plot, $V_{0}=0.8$ and $\beta=0.45$. The upper part of the boundary curve is given by equation (69). The lower part of the boundary curve is an arc of the circle of radius $\rho=1$ on the interval $\pi+\alpha \leqslant \varphi \leqslant 2 \pi-\alpha$, where $\alpha \approx 0.94 \mathrm{rad}$ corresponding to about $53^{\circ}$.

On the other hand, for a fixed $V_{0} \in\left(V_{c}, 1\right)$, the acceleration domain in figure 2 remains practically the same as $\beta \rightarrow 1 / 2$. For $\beta \rightarrow 0$, however, the shape of the domain changes significantly. In fact, for $\beta=0$, it follows from equation (69) that $\rho=\rho_{0} /|\cos \varphi|$, where $\rho_{0}$ defined by $\sqrt{3} \rho_{0}=\sqrt{1-V_{c}^{2} / V_{0}^{2}}$ is a positive constant such that $\rho_{0} \in(0,1 / \sqrt{6})$. Therefore, the acceleration domain in this case is within the intersection of the vertical strip defined by $-\rho_{0} \leqslant \hat{x} \leqslant \rho_{0}$, where $\hat{x}=\rho \cos \varphi$ and $\hat{y}=\rho \sin \varphi$, and the circle of unit radius centred at the origin of $(\hat{x}, \hat{y})$ coordinates. Though we have considered a simple circular equatorial orbit as the reference trajectory in this paper, it is not difficult to imagine that the tidal acceleration process described above-within the context of the physics of an accretion disc about the source-could possibly contribute to the formation of astrophysical jets. Moreover, tidal acceleration occurs symmetrically with respect to the equatorial plane of the Kerr source. This feature would be consistent with the observed occurrence of double jets, namely, a pair of relativistic outflows in opposite directions along the rotation axis of the central source.

\subsection{Equatorial motion}

If $y(\eta)=0$ for all $\eta$, then the motion is confined to the $(\xi, \zeta)$ plane and represents a timelike geodesic if

$\frac{1}{\Gamma^{2}}=1-\Delta_{\xi}^{2}-\Delta_{\zeta}^{2}-\left(\sigma^{2}+1\right) \xi^{2}+\zeta^{2}-4 \gamma^{2} \beta \xi\left(\xi \Delta_{\zeta}-\zeta \Delta_{\xi}\right)-\frac{1}{3} \sigma^{2}\left(\xi \Delta_{\zeta}-\zeta \Delta_{\xi}\right)^{2}>0$.

A solution of the generalized Jacobi equation (GJE) in the $(\xi, \zeta)$ plane is characterized by the parameters $\beta, V_{0}$ and $\varphi$. For $V_{0}$ extremely small compared to unity, the solution of the GJE is given essentially by the corresponding solution of the Jacobi equation as in the previous section. In general, as $V_{0}$ slowly increases towards unity, the influence of the nonlinear terms in the GJE cannot be ignored. In fact, since $\zeta$ in the Jacobi case contains a secular term, 

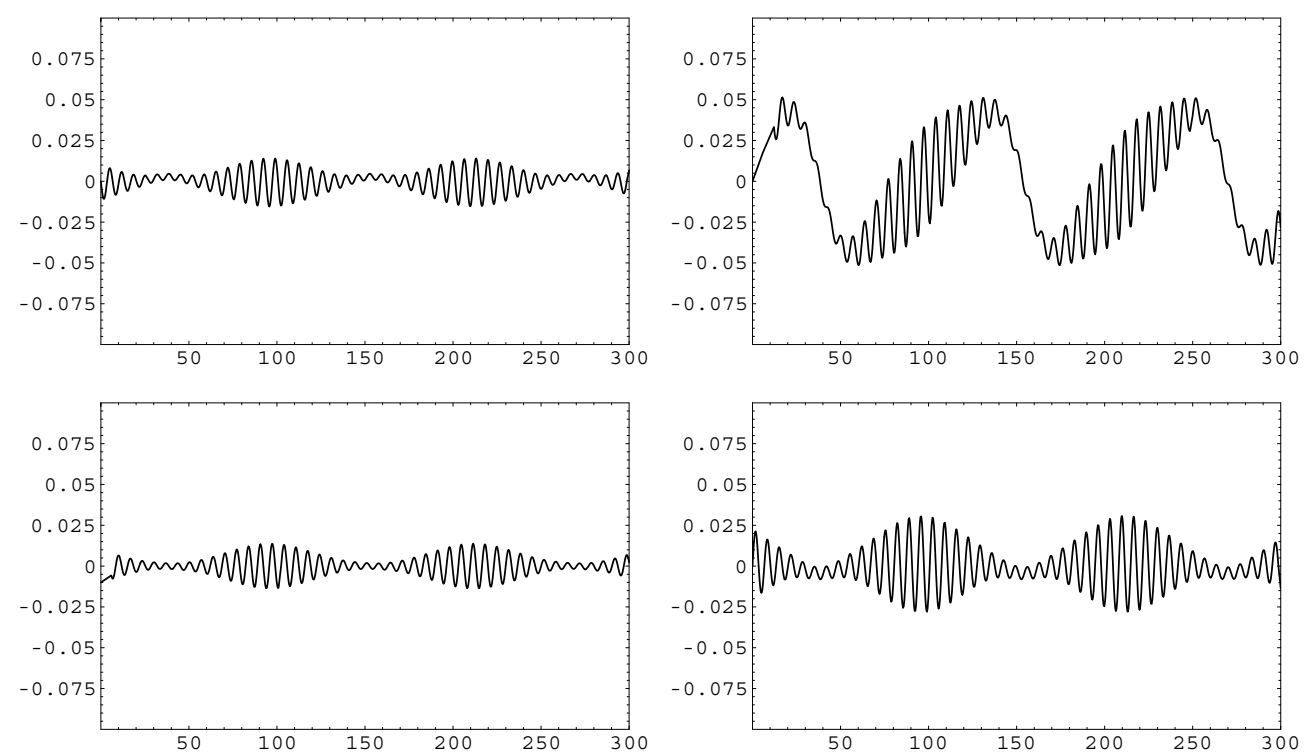

Figure 3. Plots of $(\xi, \zeta)$ versus $\eta$, top panels, and $(\dot{\xi}, \dot{\zeta})$ versus $\eta$, bottom panels, for the generalized Jacobi equation with $\beta=1 / 5$. The initial conditions are such that the motion is confined to the equatorial plane with a radially inward initial velocity such that $\vartheta=\pi / 2, \varphi=\pi$ and $V_{0}=0.01$. Note that the fast oscillation in the above panels has essentially the same frequency as in the corresponding solutions of the Jacobi equation, $\left(\xi_{J}, \zeta_{J}\right)$, given by $\xi_{J}=-\left(V_{0} / \lambda\right) \sin \lambda \eta$ and $\zeta_{J}=\left(2 V_{0} / \lambda^{2}\right)(1-\cos \lambda \eta)$.

namely, $-3(\gamma / \lambda)^{2}\left(V_{0} \sin \vartheta \sin \varphi\right) \eta$ with $\vartheta=\pi / 2$, the solution of the GJE in general grows rapidly and leaves the admissible region of the Fermi coordinate system. This difficulty can be avoided, however, in the case of the purely radial variations with $\varphi=0$ or $\pi$; in either case, the Jacobi equation has periodic solutions corresponding to the stability of the circular reference orbit under small radial perturbations. The solution of the GJE may then contain quasi-periodic oscillations exhibiting a complex beat phenomenon involving several frequencies as demonstrated in figure 3. However, such oscillations appear to occur only for $V_{0} \ll 1$, in which case equations (54)-(56) are generally not adequate physically, since higher-order tidal terms of comparable magnitude have been neglected in our analysis. Even if a quasi-periodic oscillation of the type illustrated in figure 3 survives the inclusion of relevant higher-order tidal terms, the amplitude of the effect may not be large enough to be of any physical significance in connection with the observed quasi-periodic oscillations (QPOs) [19].

\section{Discussion}

We have studied the generalized Jacobi equation for a circular reference geodesic orbit in the equatorial plane of the exterior Kerr spacetime. This equation has been reduced to an autonomous system; the corresponding tidal dynamics can then be naturally divided into vertical and equatorial motions relative to the circular orbit. In connection with vertical motion, we have clarified the role of the critical speed $V_{c}=1 / \sqrt{2}$ in the character of the solutions of the generalized Jacobi equation.

The general approach developed in this work may be of interest in connection with relativistic physics inside a space station, the tracking of artificial Earth satellites as well 
as satellite-to-satellite Doppler tracking. Moreover, in connection with the relativistic astrophysics of accretion discs around (rotating) astronomical sources, our results may be relevant for the gravitational aspects of the complex plasma physics that would be involved in the formation of high-energy jets [20]. That is, the vertical acceleration phenomenon explored in this work may be related to how relativistic jets get started above and below an accretion disc around a Kerr black hole. Once the flows are magnetically confined to regions near the axis of rotation of the central source, the results of previous investigations $[5,6,12]$ involving tidal dynamics of ultrarelativistic particles would have to be taken into account for the parallel and antiparallel flows along the Kerr rotation axis.

\section{References}

[1] Synge J L 1960 Relativity: The General Theory (Amsterdam: North-Holland)

[2] Chicone C and Mashhoon B 2002 Class. Quantum Grav. 194231

[3] Chicone C and Mashhoon B 2005 Preprint gr-qc/0511129

[4] Mashhoon B 2005 Int. J. Mod. Phys. D 142025

[5] Chicone C and Mashhoon B 2005 Ann. Phys., Lpz. 14290

Chicone C and Mashhoon B 2004 Class. Quantum Grav. 21 L139

Chicone C and Mashhoon B 2005 Class. Quantum Grav. 22195

Chicone C and Mashhoon B 2005 Astron. Astrophys. 437 L39

Chicone C and Mashhoon B 2005 Ann. Phys., Lpz. 14751

[6] Chicone C, Mashhoon B and Punsly B 2004 Int. J. Mod. Phys. D 13945

Chicone C, Mashhoon B and Punsly B 2005 Phys. Lett. A 3431

[7] Chandrasekhar S 1992 The Mathematical Theory of Black Holes (New York: Oxford University Press)

[8] Mashhoon B and Theiss D S 1982 Phys. Rev. Lett. 491542

[9] Marck J-A 1983 Proc. R. Soc. A 385431

[10] Bini D, Geralico A and Jantzen R T 2005 Class. Quantum Grav. 224729

[11] Ishii M, Shibata M and Mino Y 2005 Phys. Rev. D 71044017

[12] Kojima Y and Takami K 2006 Class. Quantum Grav. 23609

[13] Kato S, Fukue J and Mineshige S 1998 Black-Hole Accretion Disks (Kyoto: Kyoto University Press)

[14] Aliev A N, Gal'tsov D V and Petukhov V I 1986 Astrophys. Space Sci. 124137

[15] Aliev A N and Gal'tsov D V 1988 Sov. Astron. Lett. 1448

[16] Chicone C, Mashhoon B and Retzloff D G 1996 Ann. Inst. H Poincaré 6487

[17] Chicone C, Mashhoon B and Retzloff D G 1999 Helv. Phys. Acta 72123

[18] Carter B 1973 Black Holes ed C DeWitt and B S DeWitt (New York: Gordon and Breach)

[19] Strohmayer T E 2001 Astrophys. J. 552 L49

Strohmayer T E 2001 Astrophys. J. 554 L169

Wagoner R V, Silbergleit A S and Ortega-Rodriguez M 2001 Astrophys. J. 559 L25

[20] Punsly B 2001 Black Hole Gravitohydromagnetics (New York: Springer-Verlag) 